\title{
Generalized chorea as the first presentation of systemic lupus erythematosus: case report and review of literature
}

\section{Introduction}

Neuropsychiatric systemic lupus erythematosis (NPSLE) is one of the main diagnostic criteria of systemic lupus erythematosis (SLE), occurring in $25 \%-75 \%$ of affected patients. ${ }^{1,2}$ Chorea is well recognized as a NPSLE manifestation, especially in children, however it is very rare to occur as the first presenting feature of SLE. ${ }^{3}$ In this report we describe a teenage girl who presented acutely with generalized chorea and was found later on to have SLE. Her case will be described in detail and the topic of NPSLE will be reviewed.

\section{Case report}

A 14-year-old female presented with subacute onset of random, unintentional movements involving the neck, trunk, limbs as well as facial muscles. The movements were sever enough to interfere with mobility. Otherwise she did not have any systemic complaints. Had no history of a recent infectious illness and did not use any medication. Her past and family history was unremarkable. On examination, she was alert, fully oriented with intact cognitive functions. The movements were noted as involuntary, rapid and purposeless generalized movements. She could not control it and had difficulty stabilizing her head. Auscultation revealed pansystolic murmur over the apex. She had no skin lesion, joint tenderness or swelling. Blood investigations showed normochromic normocytic
Volume 8 Issue 2 - 2018

\author{
Mohamed Omer W Abdalla,' Ahmad O \\ Ahmad,' Husam A AlTahan, ' Ibrahim A \\ AlOraini, ${ }^{1,3}$ Abdulrahman M AlTahan ${ }^{1,3}$ \\ 'Dallah hospital, Saudi Arabia \\ ${ }^{2}$ King $S$ bin Abdulaziz University, Saudi Arabia \\ ${ }^{3}$ King Saud University, Saudi Arabia
}

Correspondence:Abdulrahman AlTahan, King Saud University, Saudi Arabia, Email: atahan45@hotmail.com

Received: December 29, 2017 | Published: March 13, 2018

anemia with thrombocytopenia $(\mathrm{Hb}: 8 \mathrm{~g} / \mathrm{dl}, \mathrm{MCV}: 80 \mathrm{fl}$, WBCs: $4.5 \times 10^{9}$ Platelets: $\left.84 \times 10^{9}\right)$. Had normal renal and liver functions. Coombs test was positive, ANA and anti-dsDNA Abs were positive. Furthermore her cardiolipin $\mathrm{IgG}$ was positive $(36.9 \mathrm{U} / \mathrm{ml})$ and urine analysis shows hematuria (20-30/HPF) and proteinuria $(100 \mathrm{mg} / \mathrm{dl})$. Brain MRI demonstrated multiple bilateral old and recent ischemic lesions (Figure 1). Echocardiography show mildly thickened mitral valve with prolapsed of anterior segments of mitral leaflet \& moderate mitral regurgitation.

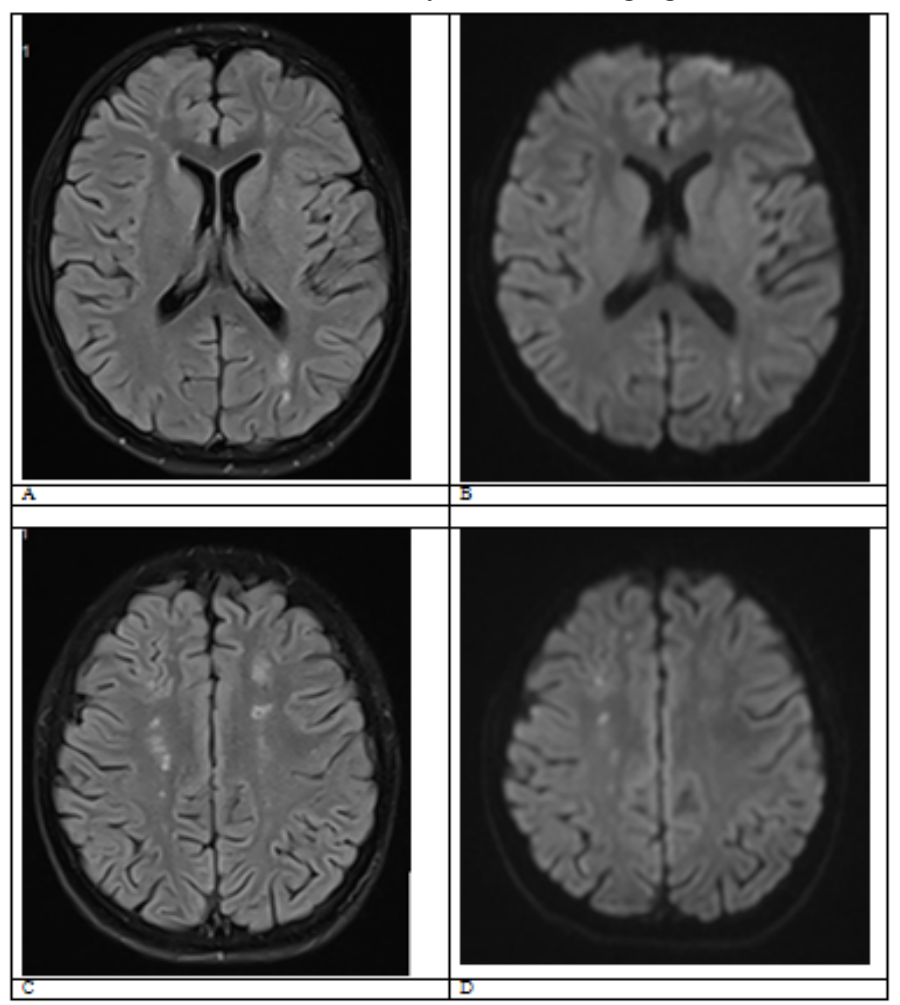

Figure I (A \& C) FLAIR and (B \& D) Diffusion-weighted images of the brain showing multiple small infarctions at different ages (old and recent) involving the deep white matter of both cerebral hemispheres. The acute infarcts show diffusion restriction on $B \& D$, while old infarctions show low signal on FLAIR images with surrounding gliosis. 
On confirming diagnosis of NPSLE, the patient was offered 3 doses of $1000 \mathrm{mg}$ IV methylprednisolone, together with enoxaparin $30 \mathrm{mg}$ BID, hydroxychloroquine 200mg BID and mycophenolic acid $1 \mathrm{~g}$ BID. She improved partially over the next few days and another course of 3 doses of methylprednisolone were offered. She showed more improvement and was able to walk unaided by the end of the week. On follow up after discharge two weeks later, involuntary movements was almost absent, and she was able to return to her normal daily routine.

\section{Discussion}

NPSLE may present before the diagnosis of SLE is made or during the course of the illness, and can be classified as either primary, when there is direct nervous system involvement, or secondary, when the involvement is a complication of the disease or its treatment. ${ }^{4,5}$

NPSLE manifestations are extremely variable and include features of both psychiatric and neurological dysfunction. ${ }^{4-9}$ Psychiatric manifestations may include cognitive dysfunction, anxiety, mood and personality disorders, and psychosis. ${ }^{5,6}$ The commonest neurological manifestations include headache, stroke, seizure, and peripheral neuropathy., ${ }^{4,-9}$ Other less common features include cranial neuropathies, transverse myelitis, meningitis, and movement disorders which occur in less than $5 \%$ of patients and include chorea, ataxia, choreoathetosis, dystonia, and hemiballismus. ${ }^{10-12}$

Chorea accounts only for about $2 \%$ of all NPSLE manifestations, which qualified it as a clinical diagnostic challenge. ${ }^{12}$ In practice, the differential diagnosis of chorea is wide and include a long list of hereditary and non-hereditary disorders. ${ }^{13-15}$ Acquired causes include drugs and toxins, Sydenham chorea, SLE and antiphospholipid (APL) syndrome, chorea gravidarum, stroke and hyperthyroidism. ${ }^{14,15}$

In view of the above described wide NPSLE clinical manifestations, pathogenesis is expected to include multiple mechanisms. However, NLSLE pathogenesis is still controversial, and probably involve immune mediated as well as ischemic mechanisms. ${ }^{16,17}$ In our patient, the old and new ischemic changes noted on MRI are consistent with an ischemic vascular process, but these can't be explained simply by the procoagulant effects of APL antibodies causing thrombotic occlusion of capillaries. It was hypothesized that an immune process may be responsible for a direct injury to the blood-brain barrier allowing autoantibodies to enter the nervous system and attack neurons involved in nigro-striatal pathways. ${ }^{16-19}$ One meta-analysis showed that NPSLE patients have higher serum levels of certain antibodies including anticardiolipin, lupus anticoagulant, anti-ribosomal $\mathrm{P}$, and antineuronal antibodies when compared with SLE patients who have no neurological involvement. ${ }^{20}$ Other secondary factors that also contribute to the pathogenesis include infections associated with immunosuppressive treatments, hypertension, and renal failure. ${ }^{21}$

NPSLE and APL syndrome are very important differential diagnosis of acute chorea, especially in children as apparently APL antibodies are more relevant in this population..$^{21} \mathrm{~A}$ higher index of suspicion should probably promote earlier diagnosis of NPSLE and particularly its most rare manifestations including chorea.

\section{Acknowledgements}

None.

\section{Conflict of interest}

The author declares no conflict of interest.

\section{References}

1. Parikh S, Swaiman KF, Kim Y. Neurologic characteristics of childhood lupus erythematosus. Pediatr Neurol. 1995;13(3):198-201.

2. Sibbitt WL Jr, Brandt JR, Johnson CR, et al. The incidence and prevalence of neuropsychiatric syndromes in pediatric onset systemic lupus erythematosus. J Rheumatol. 2002;29(7):1536-15342.

3. Cervera R, Boffa MC, Khamashta MA, et al. The Euro-Phospholipid project: epidemiology of the antiphospholipid syndrome in Europe. Lupus. 2009;18(10):889-893.

4. Wong KL, Woo EK, Yu YL, et al. Neurological manifestations of systemic lupus erythematosus: a prospective study. $Q \mathrm{~J} \mathrm{Med}$. 1991;81(294):857870 .

5. West SG. Neuropsychiatric lupus. Rheum Dis Clin North Am. 1994;20(1):129-158.

6. Hanly JG. ACR classification criteria for systemic lupus erythematosus: limitations and revisions to neuropsychiatric variables. Lupus. 2004;13(11):861-864.

7. Futrell N, Millikan C. Frequency, etiology, and prevention of stroke in patients with systemic lupus erythematosus. Stroke. 1989;20(5):583-91.

8. Appenzeller S, Cendes F, Costallat LT. Epileptic seizures in systemic lupus erythematosus. Neurology 2004;63(10):1802-12.

9. Florica B, Aghdassi E, Gladman DD, et al. Peripheral neuropathy in patients with systemic lupus erythematosus. Semin Arthritis Rheum. 2011;41(2):203-11.

10. Bertsias GK, Ioannidis JP, Aringer M, et al. EULAR recommendations for the management of systemic lupus erythematosus with neuropsychiatric manifestations: report of a task force of the EULAR standing committee for clinical affairs. Ann Rheum Dis. 2010;69(21):2074-82.

11. Joseph FG, Lammie GA, Scolding NJ. CNS lupus: a study of 41 patients. Neurology. 2007;69(7):644-54.

12. Quintero-Del-Rio AI, Van Miller. Neurologic symptoms in children with systemic lupus erythematosus. J Child Neurol. 2000;15(12):803-7.

13. Cardoso F. Difficult diagnoses in hyperkinetic disorders - a focused review. Front Neurol. 2012;3:151.

14. Walker RH. Review Differential Diagnosis of Chorea. Current neurol Neurosci Rep. 2011;11(4):385-395.

15. Bhidayasiri R, Truong DB. Review chorea and related disorders. Postgrad Med J. 2004;80(947):527-534.

16. Avcin T, Benseler SM, Tyrrell PN, et al. A followup study of antiphospholipid antibodies and associated neuropsychiatric manifestations in 137 children with systemic lupus erythematosus. Arthritis Rheum. 2008;59(2):206-213.

17. Chapman J, Rand JH, Brey RL, et al. Non-stroke neurological syndromes associated with antiphospholipid antibodies: evaluation of clinical and experimental studies. Lupus. 2003;12(7):514-517.

18. Orzechowski NM, Wolanskyj AP, Ahlskog JE, et al. Antiphospholipid antibody-associated chorea. J Rheumatol. 2008;35(11):2165-2170.

19. Ellison D, Gatter K, Heryet A, et al. Intramural platelet deposition in cerebral vasculopathy of systemic lupus erythematosus. J Clin Pathol. 1993;46(1):37-40.

20. Ho RC, Thiaghu C, Ong $\mathrm{H}$, et al. A meta-analysis of serum and cerebrospinal fluid autoantibodies in neuropsychiatric systemic lupus erythematosus. Autoimmun Rev. 2016;15(2):124-138.

21. Hanly JG, Su L, Farewell V, et al. Prospective study of neuropsychiatric events in systemic lupus erythematosus. J Rheumatol. 2009;36(7):14491459 . 